\title{
ESSENTIAL DIMENSION OF SIMPLE ALGEBRAS IN POSITIVE CHARACTERISTIC
}

\author{
SANGHOON BAEK
}

\begin{abstract}
Let $p$ be a prime integer, $1 \leq s \leq r$ integers, $F$ a field of characteristic $p$. Let $D e c_{p^{r}}$ denote the class of the tensor product of $r p$ symbols and $A g_{p^{r}, p^{s}}$ denote the class of central simple algebras of degree $p^{r}$ and exponent dividing $p^{s}$. For any integers $s<r$, we find a lower bound for the essential $p$-dimension of $A / g_{p^{r}, p^{s}}$. Furthermore, we compute upper bounds for $\operatorname{Dec}_{p^{r}}$ and $A \lg _{8,2}$ over $\operatorname{char}(F)=p$ and $\operatorname{char}(F)=2$, respectively. As a result, we show $\operatorname{ed}_{2}\left(A g_{4,2}\right)=\operatorname{ed}\left(A / g_{4,2}\right)=\operatorname{ed}_{2}\left(\mathbf{G L}_{4} / \boldsymbol{\mu}_{2}\right)=$ $\operatorname{ed}\left(\mathbf{G L}_{4} / \boldsymbol{\mu}_{2}\right)=3$ and $3 \leq \operatorname{ed}\left(A / g_{8,2}\right)=\operatorname{ed}\left(\mathbf{G L}_{8} / \boldsymbol{\mu}_{2}\right) \leq 10$ over a field of characteristic 2 .
\end{abstract}

\section{INTRODUCTION}

A numerical invariant, essential dimension of algebraic groups was introduced by Reichstein and was generalized to algebraic structures by Merkurjev. We refer to [9] for the definition of essential dimension and denote by ed and $\operatorname{ed}_{p}$ the essential dimension and essential $p$-dimension, respectively.

Let $F$ be a field, Fields / $F$ the category of field extensions over $F$, and Sets the category of sets. For every integer $n \geq 1$, a divisor $m$ of $n$ and any field extension $E / F$, let

$$
\text { Alg } g_{n, m}: \text { Fields } / F \rightarrow \text { Sets }
$$

be the functor taking a field extension $K / F$ to the set of isomorphism classes of central simple $K$-algebras of degree $n$ and exponent dividing $m$. Then, there is a natural bijection between $H^{1}\left(K, \mathbf{G L}_{n} / \boldsymbol{\mu}_{m}\right)$ and $A g_{n, m}(K)$ (see [1, Example 1.1]), thus ed $\left(A / g_{n, m}\right)=\operatorname{ed}\left(\mathbf{G L}_{n} / \boldsymbol{\mu}_{m}\right)$ and $\operatorname{ed}_{p}\left(A / g_{n, m}\right)=\operatorname{ed}_{p}\left(\mathbf{G L}_{n} / \boldsymbol{\mu}_{m}\right)$.

Let $F$ be a field of characteristic $p$. For $a \in F$ and $b \in F^{\times}$, we write $[a, b)_{p}$ for the central simple algebra over $F$ generated by $u$ and $v$ satisfying $u^{p}-u=a$, $v^{p}=b$ and $v u=u v+v$ (it is called a symbol $p$-algebra). For a field extension $E / F$, let

$$
\operatorname{Dec}_{p^{r}}: \text { Fields } / F \rightarrow \text { Sets }
$$

be the functor taking a field extension $K / F$ to the set of isomorphism classes of the tensor product of $r p$-symbols over $E$.

Some computations of the essential dimension and essential $p$-dimension of $A l g_{m, n}$ have been done. But most of them have the restriction $\operatorname{char}(F) \neq p$ on the base field $F$. In this paper, for any integers $r>s$, we find a new

The work has been supported by NSERC Discovery 385795-2010, Accelerator Supplement 396100-2010 grants, and Erhard Neher's NSERC Discovery grant. 
lower bound for $\operatorname{ed}_{p}\left(A g_{p^{r}, p^{s}}\right)$ over $\operatorname{char}(F)=p$. Moreover, we compute upper bounds for $\operatorname{Dec}_{p^{r}}$ and $A g_{8,2}$ over $\operatorname{char}(F)=p$ and $\operatorname{char}(F)=2$, respectively. As a result, we get:

Theorem 1.1. Let $F$ be a field of characteristic 2. Then

$$
\operatorname{ed}_{2}\left(A / g_{4,2}\right)=\operatorname{ed}\left(A / g_{4,2}\right)=\operatorname{ed}_{2}\left(\mathbf{G L}_{4} / \boldsymbol{\mu}_{2}\right)=\operatorname{ed}\left(\mathbf{G L}_{4} / \boldsymbol{\mu}_{2}\right)=3 .
$$

Proof. The lower bound $3 \leq \operatorname{ed}_{2}\left(A / g_{4,2}\right)$ follows from Corollary 2.4. By a Theorem of Albert, we have $\operatorname{Dec}_{4}=A / g_{4,2}$ for $p=2$, thus we get ed $\left(A / g_{4,2}\right) \leq 3$ by Proposition 3.2. As $\operatorname{ed}_{2}\left(A / g_{4,2}\right) \leq \operatorname{ed}\left(A / g_{4,2}\right)$, the result follows.

Corollary 2.4 and Corollary 3.5 give the following:

Theorem 1.2. Let $F$ be a field of characteristic 2. Then

$$
3 \leq \operatorname{ed}\left(A / g_{8,2}\right)=\operatorname{ed}\left(\mathbf{G L}_{8} / \boldsymbol{\mu}_{2}\right) \leq 10
$$

\section{LOWER BOUNDS}

Theorem 2.1. (Tsen) Let $K$ be a field of transcendental degree 1 over an algebraically closed field $F$. Then, for any central division algebra $A$ over $K$, $\operatorname{ind}(A)=\exp (A)=1$, i.e., $A=K$.

As an application of Theorem 2.1, Reichstein obtained the following result:

Corollary 2.2. 110, Lemma 9.4(a)] Let $F$ be an arbitrary field and $A$ be a division algebra of degree $n \geq 2$. Then $\operatorname{ed}(A) \geq 2$. In particular, for any integers $r, s$ and any prime $p$, $\operatorname{ed}_{p}\left(A / g_{p^{r}, p^{s}}\right) \geq 2$.

Initially, the following theorem is proved under the additional condition that $\operatorname{char}(F)$ does not divide $\exp (A)$ in [5]. In subsequent papers [6, Theorem 1.0.2] and [8, Theorem 4.2.2.3], this condition is removed:

Theorem 2.3. (de Jong) Let $K$ be a field of transcendental degree 2 over an algebraically closed field $F$. Then, for any central simple algebra $A$ over $K$, $\operatorname{ind}(A)=\exp (A)$.

As an application of Theorem 2.3, we have the following result:

Corollary 2.4. Let $F$ be an arbitrary field and $p$ be a prime. For any integers $r$ and $s$ with $s<r, \operatorname{ed}_{p}\left(A / g_{p^{r}, p^{s}}\right) \geq 3$.

Proof. By [9, Proposition 1.5], we may replace the base field $F$ by an algebraically closure of $F$. Let $K$ be a field extension of $F$ and $A$ be a central simple algebra over $K$ of $\operatorname{ind}(A)=p^{r}$ and $\exp (A) \mid p^{s}$. Let $E$ be a field extension of $K$ of degree prime to $p$. As $\operatorname{ind}(A)$ is relatively prime to $[E: K]$, we have $\operatorname{ind}\left(A_{E}\right)=\operatorname{ind}(A)=p^{r}$. Suppose that $A_{E} \simeq B \otimes E$ for some $B \in A \lg _{p^{r}, p^{s}}(L)$ and tr. $\operatorname{deg}_{F}(L)=2$. Then, by Theorem 2.3, we have $\operatorname{ind}(B)=\exp (B)$. As $p^{r}=\operatorname{ind}\left(A_{E}\right) \mid \operatorname{ind}(B)=\exp (B)$, we get $p^{r} \mid \exp (B)$. But it contradicts to $\exp (B) \mid p^{s}$. By Corollary 2.2, the result follows. 
Remark 2.5. As we see in [2, Theorem], the above lower bound 3 is much less than the best known lower bounds, but these lower bounds are valid only for $\operatorname{char}(F) \neq p$. Hence, our main application of Corollary 2.4 is for the case of $\operatorname{char}(F)=p$.

\section{UPPER BOUNDS}

\subsection{An upper bound for $\operatorname{ed}\left(D e c_{p^{r}}\right)$.}

Lemma 3.1. [3], Example 2.3 and page 298] Let $F$ be a field of characteristic $p$ and $r \geq 1$ be an integer. If $|F| \geq p^{r}$, then $\operatorname{ed}\left((\mathbb{Z} / p \mathbb{Z})^{r}\right)=1$.

Proof. From the exact exact sequence

$$
0 \rightarrow \mathbb{Z} / p \mathbb{Z} \rightarrow \mathbb{G}_{a} \stackrel{\wp}{\rightarrow} \mathbb{G}_{a} \rightarrow 0
$$

we have $H^{1}(E, \mathbb{Z} / p \mathbb{Z})=E / \wp(E)$ for any field extension $E / F$ where $\wp(x)=$ $x^{p}-x$ for $x \in E$, hence $\operatorname{ed}(\mathbb{Z} / p \mathbb{Z})=1$. By [3, Proposition 4.11], we have $\operatorname{ed}\left((\mathbb{Z} / p \mathbb{Z})^{r}\right) \geq 1$.

As $|F| \geq p^{r}$, we have an exact sequence

$$
0 \rightarrow(\mathbb{Z} / p \mathbb{Z})^{r} \rightarrow \mathbb{G}_{a} \stackrel{\wp}{\rightarrow} \mathbb{G}_{a} \rightarrow 0 .
$$

It follows from $H^{1}\left(E, \mathbb{G}_{a}\right)=0$ that $\mathbb{G}_{a}(E) \rightarrow H^{1}\left(E,(\mathbb{Z} / p \mathbb{Z})^{r}\right)$ is surjective. By [9. Proposition 1.3], we have ed $\left((\mathbb{Z} / p \mathbb{Z})^{r}\right) \leq \operatorname{dim}\left(\mathbb{G}_{a}\right)=1$.

Proposition 3.2. Let $p$ be a prime integer, $F$ be a field of characteristic $p$. If $|F| \geq p^{r}$, then $\operatorname{ed}\left(\operatorname{Dec}_{p^{r}}\right) \leq r+1$.

Proof. Let

$$
A=\otimes_{i=1}^{r}\left[a_{i}, b_{i}\right)_{p} \in \operatorname{Dec}_{p^{r}}(E)
$$

for a field extension $E / F$. As ed $\left((\mathbb{Z} / p \mathbb{Z})^{r}\right)=1$ by Lemma 3.1, there exists a sub-extension $E_{0} / F$ of $E / F$ and $c_{i} \in E_{0}$ for all $1 \leq i \leq r$ such that $c_{i} \equiv a_{i} \bmod \wp(E)$ and tr. $\operatorname{deg}_{F}\left(E_{0}\right) \leq 1$. Therefore, $A$ is defined over $L=E_{0}\left(b_{1}, \cdots, b_{r}\right)$ and tr. $\operatorname{deg}_{F}(L) \leq r+1$. Hence, ed $(A) \leq r+1$ and $\operatorname{ed}\left(\operatorname{Dec}_{p^{r}}\right) \leq r+1$.

3.2. An upper bound for $\operatorname{ed}\left(A / g_{8,2}\right)$. In this subsection we assume that the base field $F$ is of characteristic 2 . The upper bound 8 for $\operatorname{ed}\left(A / g_{8,2}\right)$ over the base field $F$ of characteristic different from 2 was determined in [1, Theorem $2.12]$. We use a similar method to find an upper bound for $\operatorname{ed}\left(A / g_{8,2}\right)$ over the base field $F$ of characteristic 2 .

For a commutative $F$-algebra $R, a \in R$ and $b \in R^{\times}$we write $[a, b)_{R}$ for the quaternion algebra $R \oplus R u \oplus R v \oplus R w$ with the multiplication table $u^{2}+u=$ $a, v^{2}=b, u v=w=v u+v$. The class of $[a, b)_{R}$ in the Brauer group $\operatorname{Br}(R)$ will be denoted by $\{a, b\}=\{a, b\}_{R}$.

Let $a \in R$ and $S=R[\alpha]:=R[t] /\left(t^{2}+t+a\right)$ with $\alpha^{2}=\alpha+a$ the quadratic extension of $R$. We write $N_{R}(a)$ for the subgroup of $R^{\times}$of all element of the form $x^{2}+x y+a y^{2}$ with $x, y \in R$, i.e., $N_{R}(a)$ is the image of the norm 
homomorphism $N_{S / R}: S^{\times} \rightarrow R^{\times}$. If $b \in N_{R}(a)$, then the quaternion algebra $[a, b)_{R}$ is isomorphic to the matrix algebra $M_{2}(R)$.

3.2.1. Rowen's construction. Rowen extended the Tignol's theorem [13] to a field of characteristic 2. We recall Rowen's argument in [11]. Let $A$ be a central simple $F$-algebra in $A g_{8,2}(F)$. By [11], there is a triquadratic splitting extension $F(\alpha, \beta, \gamma) / F$ of $A$ such that $\alpha^{2}+\alpha=a, \beta^{2}+\beta=b$, and $\gamma^{2}+\gamma=c$ for some $a, b, c \in F$. Let $L=F(\alpha)$. By [11, Corollary 5], we have

$$
\{A\}_{L}=\{b, s\}+\{c, t\}
$$

in $\operatorname{Br}(L)$ for some $s, t \in L^{\times}$.

Taking the corestriction for the extension $L / F$ in (四), we get

$$
0=2\{A\}=\left\{b, N_{L / F}(s)\right\}+\left\{c, N_{L / F}(t)\right\}
$$

in $\operatorname{Br}(F)$, hence $\left\{b, N_{L / F}(s)\right\}=\left\{c, N_{L / F}(t)\right\}$. By the chain lemma 11], Lemma 3], we have

$$
\left\{b, N_{L / F}(s)\right\}=\left\{d, N_{L / F}(s)\right\}=\left\{d, N_{L / F}(t)\right\}=\left\{c, N_{L / F}(t)\right\}
$$

in $\operatorname{Br}(F)$ for some $d \in F$. Therefore, we get $\left\{b+d, N_{L / F}(s)\right\}=\{c+$ $\left.d, N_{L / F}(t)\right\}=\left\{d, N_{L / F}(s t)\right\}=0$. By the proof of $\llbracket$, Lemma 2.3],

$$
\begin{aligned}
\{b+d, s\} & =\{b+d, k\}, \\
\{c+d, t\} & =\{c+d, l\}, \\
\{d, s t\} & =\{d, m\} .
\end{aligned}
$$

in $\operatorname{Br}(L)$ for some $k, l, m \in F^{\times}$. It follows from (1) that

$$
\{A\}_{L}=\{b+d, k\}_{L}+\{c+d, l\}_{L}+\{d, m\}_{L}
$$

in $\operatorname{Br}(L)$. Hence

$$
\{A\}=\{a, e\}+\{b, k\}+\{c, l\}+\{d, k l m\}
$$

in $\operatorname{Br}(F)$ for some $e \in F^{\times}$.

We shall need the following result:

Lemma 3.3. Let $R$ be a commutative F-algebra, $a, b \in R, T=R[\alpha]:=$ $R[t] /\left(t^{2}+t+a\right)$ and $x+y \alpha \in T^{\times}$such that $x^{2}+x y+a y^{2}=u^{2}+u v+b v^{2}$ for some $u, v \in R$. If $v+y \in R^{\times}$, then $(v+y)(x+y \alpha) \in N_{T}(b)$. In particular,

$$
\{b, x+y \alpha\}_{T}=\{b, v+y\}_{T} .
$$

Proof. The result comes from the following equality

$$
(x+y \alpha+u)^{2}+(x+y \alpha+u) v+b v^{2}=(x+y \alpha)(v+y) .
$$


3.2.2. Classifying Azumaya algebra for $A / g_{8,2}$. Consider the affine space $\mathbb{A}_{F}^{13}$ with coordinates $\mathbf{a}, \mathbf{b}, \mathbf{c}, \mathbf{d}, \mathbf{e}, \mathbf{u}, \mathbf{v}, \mathbf{w}, \mathbf{x}, \mathbf{y}, \mathbf{z}, \mathbf{m}, \mathbf{n}$ and define the rational functions:

$$
\begin{aligned}
\mathbf{f} & =\mathbf{x z}+\mathbf{w z}+\mathbf{x y} \\
\mathbf{g} & =\mathbf{w} \mathbf{y}+\mathbf{x z a}, \\
\mathbf{r} & =\left(\mathbf{g}^{2}+\mathbf{g} \mathbf{f}+\mathbf{f}^{2} \mathbf{a}+\mathbf{m}^{2}+\mathbf{m} \mathbf{n}\right) \\
\mathbf{h} & =\left(\mathbf{w}^{2}+\mathbf{w} \mathbf{x}+\mathbf{x}^{2} \mathbf{a}+1+\mathbf{u}+\mathbf{u}^{2} \mathbf{d}\right) \\
\mathbf{l} & =\left(\mathbf{y}^{2}+\mathbf{y z}+\mathbf{z}^{2} \mathbf{a}+1+\mathbf{v}+\mathbf{v}^{2} \mathbf{d}\right) \\
\mathbf{p} & =(\mathbf{u}+\mathbf{x})(\mathbf{v}+\mathbf{z})(\mathbf{n}+\mathbf{f})
\end{aligned}
$$

Let $X$ be the quasi-affine variety defined by

$$
\begin{gathered}
\mathbf{q}:=\operatorname{abcdep}\left(\mathbf{w}^{2}+\mathbf{w} \mathbf{x}+\mathbf{x}^{2} \mathbf{a}\right)\left(\mathbf{y}^{2}+\mathbf{y z}+\mathbf{z}^{2} \mathbf{a}\right)\left(\mathbf{g}^{2}+\mathbf{g f}+\mathbf{f}^{2} \mathbf{a}\right) \neq 0, \\
\mathbf{b u} \mathbf{u}^{2}=\mathbf{h}, \mathbf{c v}^{2}=\mathbf{l}, \mathbf{d n}^{2}=\mathbf{r}
\end{gathered}
$$

i.e., $X=\operatorname{Spec}(R)$ with

$R=F\left[\mathbf{a}, \mathbf{b}, \mathbf{c}, \mathbf{d}, \mathbf{e}, \mathbf{u}, \mathbf{v}, \mathbf{w}, \mathbf{x}, \mathbf{y}, \mathbf{z}, \mathbf{m}, \mathbf{n}, \mathbf{q}^{-1}\right] /<\mathbf{b u}^{2}+\mathbf{h}, \mathbf{c v}^{2}+\mathbf{l}, \mathbf{d n}^{2}+\mathbf{r}>$.

Let $T=R[\alpha]$ and $S=R[\alpha, \beta, \gamma]$ with $\alpha^{2}=\alpha+\mathbf{a}, \beta^{2}=\beta+\mathbf{b}, \gamma^{2}=\gamma+\mathbf{c}$. Consider the Azumaya $R$-algebra

$$
\mathcal{B}^{\prime}=[\mathbf{a}, \mathbf{e})_{R} \otimes[\mathbf{b}, \mathbf{x}+\mathbf{u})_{R} \otimes[\mathbf{c}, \mathbf{z}+\mathbf{v})_{R} \otimes[\mathbf{d}, \mathbf{p})_{R} .
$$

By Lemma 3.3, we get the followings :

$$
\begin{aligned}
(\mathbf{x}+\mathbf{u})(\mathbf{w}+\mathbf{x} \alpha) & \in N_{T}(\mathbf{b}+\mathbf{d}) \subset N_{S}(\mathbf{d}), \\
(\mathbf{z}+\mathbf{v})(\mathbf{y}+\mathbf{z} \alpha) & \in N_{T}(\mathbf{c}+\mathbf{d}) \subset N_{S}(\mathbf{d}), \\
(\mathbf{n}+\mathbf{f})(\mathbf{w}+\mathbf{x} \alpha)(\mathbf{y}+\mathbf{z} \alpha) & \in N_{T}(\mathbf{d}) \subset N_{S}(\mathbf{d}) .
\end{aligned}
$$

It follows from (2) that

$$
\left\{\mathcal{B}^{\prime}\right\}_{T}=\{\mathbf{b}, \mathbf{w}+\mathbf{x} \alpha\}+\{\mathbf{c}, \mathbf{y}+\mathbf{z} \alpha\}
$$

in $\operatorname{Br}(T)$.

Since $\mathbf{p} \in N_{S}(\mathbf{d}),[\mathbf{d}, \mathbf{p})_{S}$ is isomorphic to the matrix algebra $M_{2}(S)$. In particular,

$$
M_{2}(R) \subset M_{2}(S) \simeq[\mathbf{d}, \mathbf{p})_{S} \subset \mathcal{B}^{\prime}
$$

and hence $\mathcal{B}^{\prime} \simeq M_{2}(\mathcal{B})$ for the centralizer $\mathcal{B}$ of $M_{2}(R)$ in $\mathcal{B}^{\prime}$ by the proof of [7, Theorem 4.4.2]. Then $\mathcal{B}$ is an Azumaya $R$-algebra of degree 8 that is Brauer equivalent to $\mathcal{B}^{\prime}$ by [12, Theorem 3.10].

Proposition 3.4. The Azumaya algebra $\mathcal{B}$ is classifying for $A g_{8,2}$, i.e, the corresponding $\mathbf{G L}_{8} / \boldsymbol{\mu}_{2}$-torsor over $X$ is classifying. 
Proof. Let $A \in A \lg _{8,2}(K)$, where $K$ is a field extension of $F$. We shall find a point $p \in X(K)$ such that $A \simeq \mathcal{B}(p)$, where $\mathcal{B}(p):=\mathcal{B} \otimes_{R} K$ with the $F$-algebra homomorphism $R \rightarrow K$ given by the point $p$.

Following Rowen's construction, there is a triquadratic splitting extension $K(\alpha, \beta, \gamma) / K$ of $A$ such that $\alpha^{2}+\alpha=a, \beta^{2}+\beta=b$, and $\gamma^{2}+\gamma=c$ for some $a, b, c \in K$. Let $L=K(\alpha)$, so

$$
\{A\}_{L}=\{b, s\}+\{c, t\}
$$

in $\operatorname{Br}(L)$ for some $s=w+x \alpha$, and $t=y+z \alpha \in L^{\times}$. We have

$\left\{b, w^{2}+w x+x^{2} a\right\}=\left\{d, w^{2}+w x+x^{2} a\right\}=\left\{d, y^{2}+y z+z^{2} a\right\}=\left\{c, y^{2}+y z+z^{2} a\right\}$

in $\operatorname{Br}(K)$ for some $d \in K$, so $\left\{b+d, w^{2}+w x+x^{2} a\right\}=\left\{c+d, y^{2}+y z+z^{2} a\right\}=$ $\left\{d,\left(w^{2}+w x+x^{2} a\right)\left(y^{2}+y z+z^{2} a\right)\right\}=0$. Hence

$$
\begin{aligned}
w^{2}+w x+x^{2} a & =u^{\prime 2}+u^{\prime} u+u^{2}(b+d), \\
y^{2}+y z+z^{2} a & =v^{\prime 2}+v^{\prime} u+v^{2}(c+d), \\
\left(w^{2}+w x+x^{2} a\right)\left(y^{2}+y z+z^{2} a\right) & =m^{2}+m n+n^{2} d
\end{aligned}
$$

for some $u, u^{\prime}, v, v^{\prime}, m, n$ in $K$. Moreover, we may assume that $u^{\prime} \neq 0$. Replacing $w, x$ and $u$ by $w u^{\prime}, x u^{\prime}$ and $u^{\prime} u$ respectively, we may assume that $u^{\prime}=1$. Similarly, we may assume that $v^{\prime}=1$.

We also may assume that $u \neq x$ by replacing $u$ by $u /(b+d)$. Similarly, we may assume that $v \neq z$ and $n+x z+w z+x y \neq 0$. It follows from Lemma 3.3 that

$$
\begin{aligned}
\{b+d, w+x \alpha\} & =\{b+d, u+x\}_{L}, \\
\{c+d, y+z \alpha\} & =\{c+d, z+v\}_{L}, \\
\{d,(w+x \alpha)(y+z \alpha)\} & =\{d, n+x z+w z+x y\}_{L}
\end{aligned}
$$

in $\operatorname{Br}(L)$. Hence

$\{A\}=\{a, e\}+\{b, u+x\}+\{c, z+v\}+\{d,(u+x)(z+v)(n+x z+w z+x y)\}$

in $\operatorname{Br}(K)$ for some $e \in K^{\times}$.

Let $p$ be the point $(a, b, c, d, e, u, v, w, x, y, z, m, n)$ in $X(K)$. We have $\{\mathcal{B}(p)\}=$ $\{A\}$ and hence $\mathcal{B}(p) \simeq A$ as $\mathcal{B}(p)$ and $A$ have the same dimension.

Corollary 3.5. $\operatorname{ed}\left(A / g_{8,2}\right) \leq 10$.

Proof. There is surjective morphism $X \rightarrow A / g_{8,2}$ by Proposition 3.4. By [9, Proposition 1.3], $\operatorname{ed}\left(A / g_{8,2}\right) \leq \operatorname{dim}(X)=10$.

\section{REFERENCES}

[1] S. Baek and A. Merkurjev, Invariants of simple algebras, Manuscripta Math, Vol. 129, No. 4 (2009), 409-421.

[2] S. Baek and A. Merkurjev, Essential dimension of central simple algebras, to appear in Acta Math. 
[3] G. Berhuy and G. Favi, Essential dimension: a functorial point of view (after A. Merkurjev), Doc. Math. 8 (2003), 279-330 (electronic).

[4] H. Dherte, Quadratic descent of involutions in degree 2 and 4, Proc. Amer. Math. Soc. 123 (1995), no. 7, 1963-1969.

[5] A. J. de Jong, The period-index problem for the Brauer group of an algebraic surface, Duke Math. J. 123 (2004), 71-94.

[6] A. J. de Jong and J. Starr, Almost proper GIT-stacks and discriminant avoidance, http://www.math.columbia.edu/ dejong/papers/5-torsor.pdf.

[7] I. N. Herstein, Noncommutative rings, Carus Mathematical Monographs, vol. 15, Mathematical Association of America, Washington, DC, 1994, Reprint of the 1968 original, With an afterword by L. W. Small.

[8] M. Lieblich, Twisted sheaves and the period-index problem, Compos. Math. 144 (2008), no. $1,1-31$.

[9] A. S. Merkurjev, Essential dimension, Quadratic forms - algebra, arithmetic, and geometry, Contemp. Math., vol. 493, Amer. Math. Soc., Providence, RI, (2009), pp. 299-325.

[10] Z. Reichstein, On the notion of essential dimension for algebraic groups, Transform. Groups 5 (2000), no. 3, 265-304.

[11] L. Rowen, Division algebras of exponent 2 and characteristic 2, J. Algebra 90 (1984), no. $1,71-83$.

[12] D. J. Saltman, Lectures on division algebras, CBMS Regional Conference Series in Mathematics, vol. 94, American Mathematical Society, Providence, RI, 1999.

[13] J.-P. Tignol, Sur les classes de similitude de corps à involution de degré 8, C. R. Acad. Sci. Paris Sér. A-B 286 (1978), no. 20, A875-A876.

Department of Mathematics and Statistics, University of Ottawa, Canada

E-mail address: sbaek@uottawa.ca 